\title{
RAPID HARD X-RAY VARIABILITY IN GRO J0422+32
}

F. VAN DER HOOFT, J. VAN PARADIJS AND M. VAN DER KLIS

University of Amsterdam, The Netherlands

C. KOUVELIOTOU, D.J. CRARY AND M.H. FINGER

USRA and NASA/MSFC, USA

B.C. RUBIN

RIKEN Institute, Japan

B.A. HARMON AND G.J. FISHMAN

NASA/MSFC, USA

AND

W.H.G. LEWIN

$M I T, U S A$

\section{Introduction}

The soft X-ray transient (SXT) GRO J0422+32 (Nova Persei 1992) was detected with the Burst And Transient Source Experiment (BATSE) on board the CGRO on 1992 August 5 (Paciesas et al. 1992) (Truncated Julian Day [TJD] 8839). The source intensity of GRO J0422+32 increased rapidly, reaching a flux of $\sim 3 \mathrm{Crab}(40-230 \mathrm{keV})$ within days after its first detection (Harmon et al. 1992). Hereafter, the X-ray intensity of the source decreased exponentially with a decay time of $\sim 43$ days (Vikhlinin et al. 1995). A secondary maximum of the X-ray intensity was reached at TJD 8978, 139 days after the first detection of the source. The daily averaged flux history of GRO J0422+32 in the 40-150 keV energy band is presented in Figure 1.

\section{Time series analysis}

For our analysis, we considered uninterrupted data segments of 512 successive time bins (of $1.024 \mathrm{~s}$ each) on which we performed Fast Fourier Transforms (FFTs) covering the frequency interval $0.002-0.488 \mathrm{~Hz}$. Per day, we obtained typically 35 of such segments while the source was above 

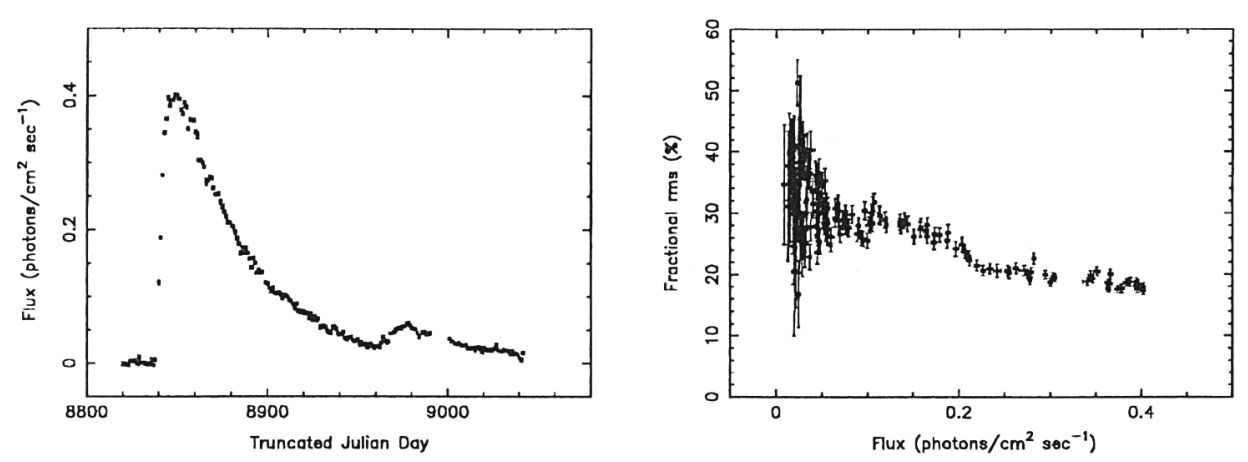

Figure 1. (Left:) Daily averaged flux history of GRO J0422+32 in the $40-150 \mathrm{keV}$ energy band, as determined using the Earth occultation technique. The first detection of the source was on TJD 8839 (1992 August 5). (Right:) The fractional rms amplitudes (20-100 $\mathrm{keV}$ ) determined in the interval $0.01-0.48 \mathrm{~Hz}$, plotted versus the flux of GRO J0422+32 in the $40-150 \mathrm{keV}$ energy band.

the Earth horizon. For each data segment and for each of the eight detectors separately, we calculated and coherently summed the FFTs of the lowest two energy channels $(20-50,50-100 \mathrm{keV})$. For those detectors which had the source within 60 degrees of the normal, these FFTs were again coherently summed (weighted by the ratio of the source to the total count rates) and converted to Power Density Spectra (PDSs). The PDSs were normalized such that the power density is given in units of $(\mathrm{rms} / \mathrm{mean})^{2}$ $\mathrm{Hz}^{-1}$. Our analysis fully covers the X-ray outburst of GRO J0422+32.

We determined fractional rms amplitudes by integrating the single-day averaged PDSs of GRO J0422+32 over the frequency interval $0.01-0.48 \mathrm{~Hz}$. During the rise to the first X-ray maximum the fractional rms amplitudes decrease monotonically, while gradually increasing during the exponential decline of the light curve. The largest fractional rms amplitudes were obtained near TJD 8960, short y before the onset of the secondary maximum in X-rays. At the secondary rnaximum, the fractional rms amplitudes again reach a local minimum. In Figure 1 we present the fractional rms amplitudes $(20-100 \mathrm{keV}, 0.01-0.48 \mathrm{~Hz})$, plotted versus the $40-150 \mathrm{keV}$ flux of GRO J0422+32. At low flux levels the fractional rms amplitudes become uncertain and are dominated by detector noise due to unresolved sources in the uncollimated field of view of BATSE. At higher flux levels the fractional rms amplitude and flux appear to be anti-correlated.

\section{References}

Harmon, B.A. et al., 1992, IAUC, 5584

Paciesas, W.S. et al. 1992, IAUC, 5580

Vikhlinin, A. et al. 1995, ApJ, 441, 779 